\title{
COMUNICACIÓN
}

\section{Hallazgos Clínicos y Tomográficos en un Ovino con Alteraciones Nerviosas: Reporte de un Caso}

\author{
Clinical and Tomographic Findings in a Ram with Signs of Nervous \\ Disorders: A CASE REPORT
}

Rocío Sandoval M. ${ }^{1,2}$, Luis Ruiz G. ${ }^{1}$, Alfredo Delgado C. ${ }^{1}$, Joe Pizarro P. ${ }^{1}$

\section{Resumen}

Se presentan los hallazgos clínicos y tomográficos de un carnero reproductor de 3.5 años, de raza Corriedale, con signos de alteraciones nerviosas. Al examen físico presentó signos de depresión, postura anormal, ataxia y paresia facial derecha, producto de un síndrome de hemisferio izquierdo. El examen radiográfico no reveló alteraciones, pero en la tomografía axial computarizada craneal se halló una masa hipodensa bilobulada de 6.4 x $3.4 \mathrm{~cm}$. de diámetro antero posterior por transverso, localizada en el lado izquierdo, desde la región frontal hasta la región occipital. El parénquima residual estaba desplazado en sentido periférico y con alteración de la densidad, sin poderse diferenciar sustancia gris de sustancia blanca, las cavidades ventriculares se encontraban colapsadas, la fosa posterior mostraba una depresión del cerebelo, y los espacios epidural y subdural se encontraban disminuidos. El diagnóstico anatomopatológico fue lesión tumoral hipodensa en el hemisferio cerebral izquierdo que cruza la línea media y asocia signos de hipertensión endocraneana compatible con un glioblastoma.

Palabras clave: tumor cerebral, ovino, alteraciones nerviosas, tomografía axial computarizada

\section{Abstract}

This report describes the clinical and computed tomography findings in a stud Corriedale ram, 3.5 years old, with signs of nervous disorders. Physical examination showed signs of depression, abnormal posture, ataxia, right facial paresis, due to a left hemisphere syndrome. X-ray examination revealed no abnormalities, but a cranial computed

\footnotetext{
${ }^{1}$ Clínica de Animales Mayores, Facultad de Medicina Veterinaria, Universidad Nacional Mayor de San Marcos, Lima, Perú

${ }^{2}$ E-mail: rociossandovalm@gmail.com
}

Recibido: 25 de abril de 2014

Aceptado para publicación: 30 de agosto de 2014 
tomography scan showed a hypodense bilobed mass of $6.4 \times 3.4 \mathrm{~cm}$ anteroposterior by transverse diameters, located from the front to the occipital region of the left side. The residual parenchyma was shifted towards the periphery with alteration of the density, unable to differentiate the gray matter from the white matter, the ventricular cavities were collapsed, the posterior fossa showed a depression exerted by the cerebellar lesion, and the epidural and subdural spaces were diminished. The pathological diagnosis was hypodense tumoral lesion in the left cerebral hemisphere crossing the midline with signs of intracranial hypertension compatible with glioblastoma.

Key words: brain tumor, sheep, nervous disorders, computerized axial tomography

\section{INTRODUCCIÓN}

El objetivo de la exploración neurológica completa es identificar la presencia de signos clínicos asociados con el sistema nervioso (Bagley y Mayhew, 2002; Jackson y Cockcroft, 2002). Esto, sin embargo, requiere examinar todos los órganos y sistemas del cuerpo, dado que muchas alteraciones nerviosas son productos de enfermedades que no solo afectan el sistema nervioso (Hindson y Winter, 2002); de allí la importancia del diagnóstico diferencial (Jackson y Cockcroft, 2002).

En el ovino, las alteraciones neurológicas más comunes son la toxemia de la gestación y la coenurosis (Hindson y Winter, 2002); sin embargo, hay diversas alteraciones neurológicas que son causadas por enfermedades metabólicas como la hipomagnesemia, exotoxinas bacterianas como el tétano, enfermedades parasitarias como oestrosis (Suárez et al., 2011), venenos químicos como el plomo (Bergqvist et al., 1991), venenos de plantas como la campanilla morada (Ipomoea carnea) (Sabogal y Borkowski, 2007), infecciones bacterianas como la listeriosis, lesiones traumáticas como el daño cervical (Hindson y Winter, 2002), trastornos degenerativos como scrapie (Farías et al., 2011), y neoplasias como el oligodendroglioma (Derakhshanfar y Mozaffari, 2010).
La tomografía axial computarizada es una técnica diagnóstica que ha tomado mayor relevancia en la medicina veterinaria en el Perú (Donaires et al., 2013). La tomografía computarizada de cerebro puede ayudar a descartar la posibilidad de que los signos neurológicos sean el resultado de problemas tales como quistes parasitarios o abscesos (Gómez et al., 2007); sin embargo, el costo de la técnica es un factor limitante.

El presente trabajo tuvo como objetivo presentar los hallazgos clínicos y tomográficos de un ovino con signos de alteraciones nerviosas.

\section{Caso Clínico}

Un carnero Corriedale de 3.5 años de edad, fue remitido a la Clínica de Animales Mayores de la Facultad de Medicina Veterinaria, Universidad Nacional Mayor de San Marcos, Lima. El carnero formaba parte del plantel de reproductores de la SAIS Pachacútec, ubicada en el distrito de Marcapomacocha, provincia de Yauli, Junín, Perú.

El animal estuvo manifestando torneo, decaimiento e inapetencia por dos semanas. El diagnóstico inicial fue de coenurosis cerebral, considerando la presencia de perros pastores en la zona. El animal, dada su condición 
Cuadro 1. Exploración neurológica de un carnero reproductor Corriedale de 3.5 años de edad con historia de torneo, decaimiento e inapetencia

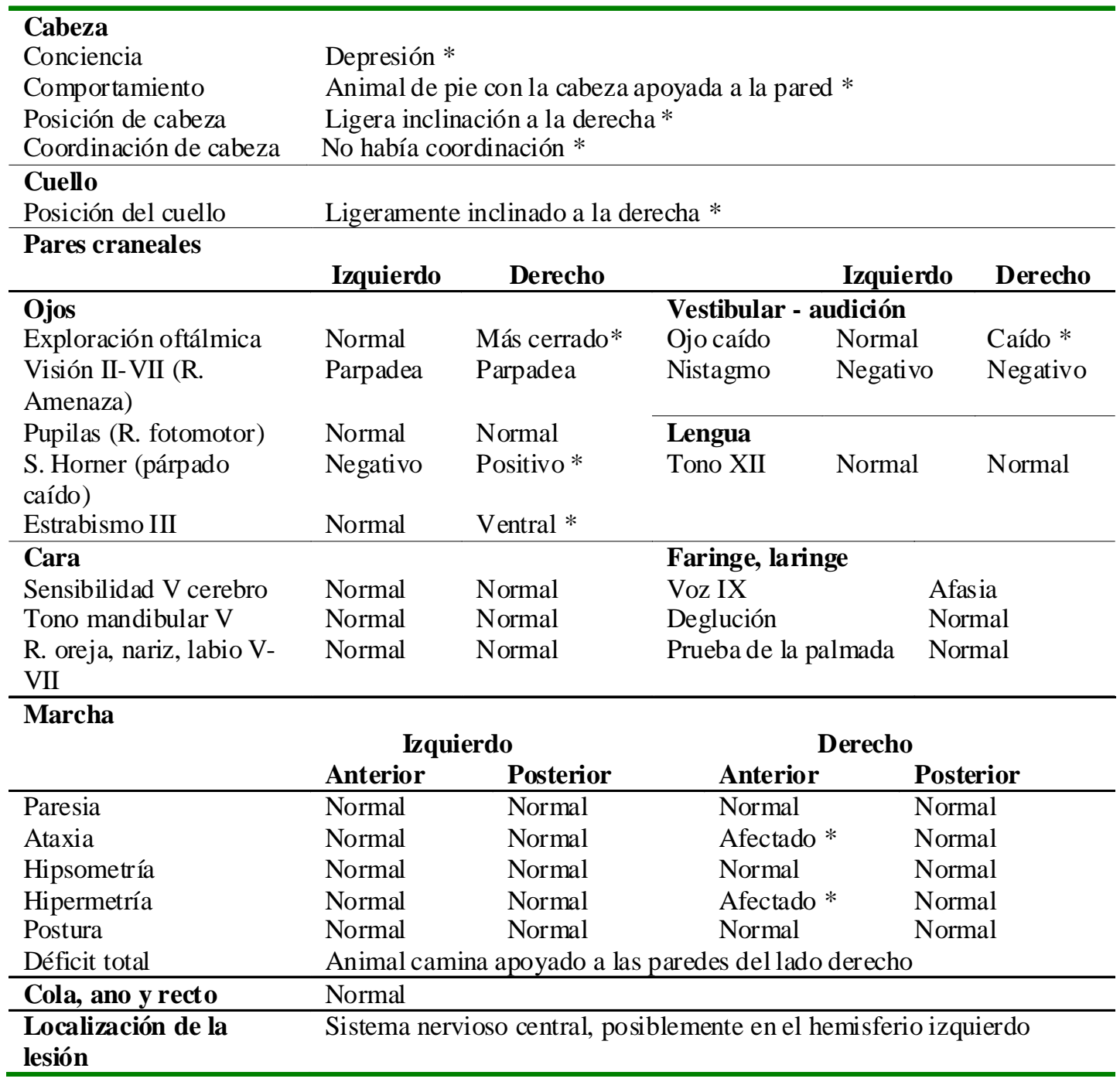

* Indica alteración

de reproductor y alto valor genético, fue remitido a la clínica para la extirpación quirúrgica del quiste parasitario.

El animal fue internado y se le dejó en reposo hasta el día siguiente. A la inspección general, el carnero mostraba signos de ataxia, caminaba apoyándose en las paredes y sobre el lado derecho. Además, tenía el cuello girado hacia ese mismo lado y la cabeza inclinada, y presentaba disnea y frecuencia respiratoria incrementada. $\mathrm{Al}$ examen clínico no presentaba fiebre $\left(38.7^{\circ} \mathrm{C}\right.$; valor normal [VN]: $\left.39-40{ }^{\circ} \mathrm{C}\right)$, pero los movimientos ruminales estaban disminuidos ( 3 en 5 min; $\mathrm{VN}$ : $5-15 / 5 \mathrm{~min})$, frecuencia respiratoria incrementada (120 por minuto; VN: 12-72), frecuencia cardiaca normal (60 latidos por minuto; VN: 60-120) (Smith y George, 2010). 

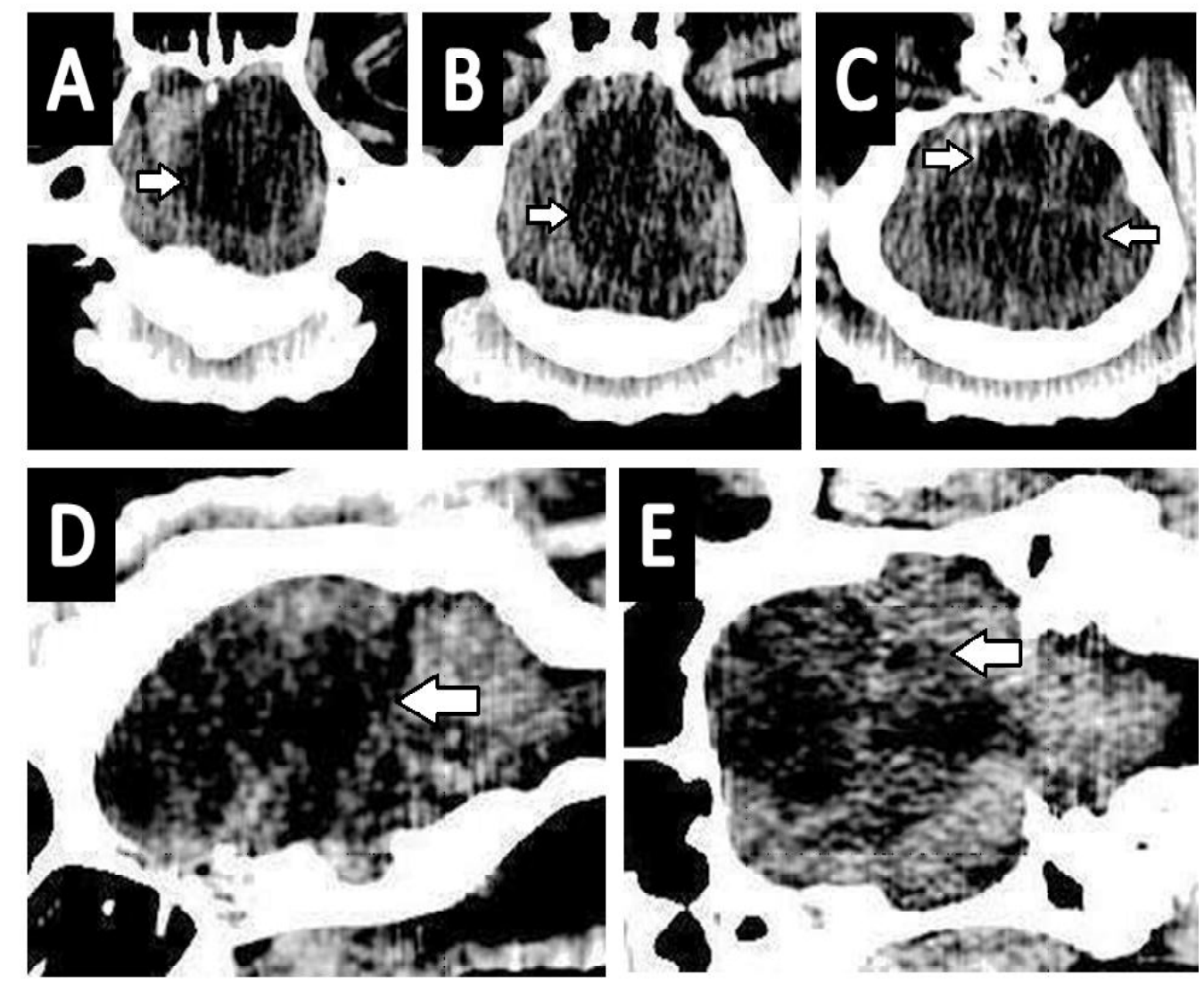

Figura 1. Imágenes tomográficas de un carnero reproductor Corriedale 3.5 años de edad con historia de torneo, decaimiento e inapetencia. (A, B, C) Cortes coronales del cráneo de antero a posterior mostrando una masa hipodensa bilobulada en el hemisferio izquierdo que cruza la línea media. (D) Corte sagital del cráneo mostrando una lesión hipodensa de $6.4 \mathrm{~cm}$ de diámetro antero posterior. (E) Corte axial del cráneo mostrando una lesión hipodensa bilobulada de $3.4 \mathrm{~cm}$ diámetro transverso (flechas blancas indican la ubicación de la lesión).

Se procedió a realizar un examen físico del sistema nervioso (Bagley y Mayhew, 2002). Los hallazgos se describen en el Cuadro 1. La depresión encontrada en el estado de conciencia y el comportamiento anormal, que consistía en apoyar la cabeza a un muro y la ataxia, son signos compatibles con un daño en los hemisferios cerebrales. Asimismo, el ojo derecho más cerrado, el síndrome de Horner positivo, el estrabismo derecho ventral y la afasia son indicativos de una lesión cerebral del hemisferio izquierdo; por lo que el diagnóstico anatomopatólogico fue paresia derecha, producto de un síndrome de hemisferio izquierdo.

Se hicieron exámenes auxiliares para obtener mayor información del caso. Se realizó un hemograma y pruebas bioquímicas (medición de lactato deshidrogenasa [LDH], un marcador general, y medición de creatinina quinasa $[\mathrm{CK}]$, un marcador más específico del daño cerebral). Los valores del hemograma estuvieron dentro de los rangos 
normales; sin embargo, se encontró un incremento de la CK (599 UI/L; VN: 100-547), lo cual confirma un daño celular localizado en el encéfalo.

Se procedió a realizar pruebas radiográficas (General Electric, Mobile 225, EEUU). El animal fue medicado con xilacina $(0.1 \mathrm{mg} / \mathrm{kg})$ y atropina $(0.02 \mathrm{mg} / \mathrm{kg})$ por vía intramuscular. Se indujo sedación completa mediante diazepam $(0.5 \mathrm{mg} / \mathrm{kg})$. Se le colocó en posición decúbito esternal y se tomaron dos placas radiográficas del cráneo, una en posición latero-lateral izquierda y otra en posición dorso-ventral, ambas con $70 \mathrm{kvp}, 100$ $\mathrm{mA}, 1 / 15$. El estudio radiográfico no reveló alteraciones.

Luego del examen radiográfico, se procedió a realizar una tomografía axial computarizada del cráneo en un centro especializado por imágenes, utilizando un equipo SOMATOM Spirit ${ }^{\circ}$ (Siemens, Alemania). Se volvió a sedar al animal con el mismo procedimiento, y se le colocó en posición decúbito dorsal con las extremidades torácicas extendidas cranealmente y las pélvicas extendidas caudalmente. La cabeza fue fijada al soporte metálico de la camilla. Imágenes transversales fueron obtenidas a nivel de cráneo, desde la lámina cribosa del etmoides hasta el foramen magno, mediante una radiografía digital lateral inicial (topograma). El estudio tomográfico fue realizado sin la administración de contraste y graficado con cortes de 5 $\mathrm{mm}$ en ventana parenquimal.

Los hallazgos del examen tomográfico se muestran en la Fig. 1. El examen evidenció surcos, cisuras y circunvoluciones cerebrales de aspecto disminuido. El parénquima cerebral mostró una lesión hipodensa bilobulada de $6.4 \times 3.4 \mathrm{~cm}$ de diámetro antero posterior por transverso, localizada desde la región frontal hasta la región occipital en el lado izquierdo, colapsando completamente los ventrículos. El parénquima residual se encontró desplazado en sentido periférico y con alteración de la densidad, sin poderse diferenciar la sustancia gris de la sustancia blanca.
Los ganglios basales fueron de difícil evaluación. Las cavidades ventriculares estaban colapsadas, la fosa posterior mostró una depresión del cerebelo ejercida por la lesión descrita, y los espacios epidural y subdural se encontraron disminuidos.

Con base a estos hallazgos, el diagnóstico anatomopatológico fue lesión tumoral hipodensa en el hemisferio cerebral izquierdo que cruza la línea media y asocia signos de hipertensión endocraneana. El pronóstico del paciente era malo y con una limitada probabilidad de supervivencia; sin embargo, a solicitud de los propietarios, se recomendó un tratamiento paliativo a base de altas dosis de corticoides.

\section{Discusión}

El encéfalo, para su estudio, se divide en estructuras supratentoriales e infratentoriales (Bagley y Mayhew, 2002). El examen clínico evidenció que se encuentra afectada principalmente el área supratentorial, lo cual fue confirmado por el estudio tomográfico que evidenció una lesión tumoral en el cerebro (Fig. 1). Las funciones neurológicas que se encontraron afectadas en el carnero indican que el daño se encontraba distribuido en el encéfalo, incluyendo los lóbulos del cerebro y cerebelo (Chandana et al., 2008).

Los signos clínicos de los tumores encefálicos varían con la localización. En el presente caso hubo alteraciones de la marcha (ataxia, paresia, dismetría), paresia facial, giro lateral de la nuca y estrabismo entre otros (Cuadro 1), los cuales concuerdan con los signos clínicos reportados por Smith y George (2010). Tumores primarios del sistema nervioso han sido descritos en los rumiantes (Braun et al., 2005; Derakhshanfar y Mozaffari, 2010); sin embargo, dada su localización, se pueden descartar tumores que provengan de una localización infratentorial, como un meduloblastoma o un epedimoblastoma (Martínez et al., 2008). 
La amplia distribución y extensión de la lesión y la evolución clínica acelerada indican que se trata posiblemente de un caso de un tumor cerebral grado IV, con signos de crecimiento rápido e invasivo (Chandana et al., 2008). Además, las características tomográficas de hipodensidad, bordes no definidos y ausencia de zonas de calcificación, hacen sospechar de un glioblastoma (Jacobs et al., 2005), el cual es un tumor de los astrocitos (Jacobs et al., 2005; Chandana et $a l .$, 2008). En humanos, el glioblastoma grado IV es el tumor cerebral primario más mortal y común. A pesar de una estrategia de tratamiento multimodal agresiva, la mediana de supervivencia de los pacientes con gliomas es limitada, dependiendo del grado y la edad al momento del diagnóstico (Jacobs et al., 2005).

\section{Literatura Citada}

1. Bagley RS, Mayhew IG. 2002. Exploración clínica del sistema nervioso. En: Radostits OM, Mayhew IG, Houston DM (eds). Examen y diagnóstico clínico en veterinaria. España: Elsevier Science. $p$ 493-542.

2. Bergqvist E, Parada R, Palavicino I. 1991. Ingestión critica de metales pesados en diversas especies animales. Agric Téc 51: 370-373.

3. Braun $U$, Hilbe $M$, Ehrensperger $F$. 2005. Clinical and pathological findings in a goat with cerebral gliomatosis. Vet $\mathbf{J}$ 170: 381-383. doi: 10.1016/ j.tvjl.2004.09.010

4. Chandana SR, Movva S, Arora M, Singh T. 2008. Primary brain tumors in adults. Am Fam Physician 77: 1423-1430.

5. Derakhshanfar A, Mozaffari AA. 2010. First report of oligodendroglioma in a sheep. J S Afr Vet Assoc 81: 114-115.

6. Donaires $R$, Díaz D, Chipayo Y, Gavidia C. 2013. Comparación de la radiografía simple y la tomografía computarizada en el diagnóstico de hernia discal tipo 1 en perros. Rev Inv Vet Perú 24: 78-85.
7. Farías G, Garces $H$, Larenas $J$, Ramírez A, Lecocq C. 2011. Enfermedades producidas por priones en los animales. Avances en Ciencias Veterinarias 26 [Internet]. Disponible en: http:// www.avances veterinaria.uchile.cl/ index.php/ACV/article/view/17527/ 18936

8. Gómez M, Tadich $N$, Mieres $M$, Bustamante H, Galecio J, Herve M. 2007. Hallazgos de tomografía computarizada en coenurosis cerebral crónica con hidrocéfalo secundario en una oveja joven. Arch Med Vet 39: 281-285. doi:10.4067/ S0301-732X2007000300013

9. Hindson JC, Winter AC. 2002. Manual of sheep diseases. $2^{\text {nd }}$ ed. Oxford: Blackwell Science. 289 p.

10. Jackson P, Cockcroft P. 2002. Clinical examination of farm animals. Oxford: Blackwell Science. 313 p.

11. Jacobs AH, Kracht LW, Gossmann A, Rüger MA, Thomas AV, Thiel A, Herholz K. 2005. Imaging in neurooncology. NeuroRX 2: 333-347. doi: 10.1602/neurorx.2.2.333

12. Martínez J, García A, Garaizar C. 2008. Tumores cerebrales infantiles: diagnóstico y semiología neurológica. En: Narbona J, Casas C (eds). Protocolos diagnóstico terapeúticos de la AEP: neurología pediátrica. $2^{\mathrm{a}}$ ed. España: AEP. $\mathrm{p}$ 203-209.

13. Sabogal A, Borkowski D. 2007. Estado actual de la investigación sobre Ipomoea carnea: toxicidad en ganado caprino. Rev Quím 21:29-35.

14. Smith MO, George LW. 2010. Enfermedades del sistema nervioso. En: Smith BP (ed). Medicina interna de grandes animales. $4^{\mathrm{a}}$ ed. España: Elsevier Science. p 972-1111.

15. Suarez VH, Busetti MR, Real Ortellado M. 2011. Prevalencia de enfermedades y manejo sanitario en los sistemas de producción ovina de lana y carne de La Pampa, Argentina. Rev Vet Arg 28. [Internet]. Disponible en: http:// www.veterinariargentina.com/revista/ 2011/12/page/5/ 